IOS Press

\title{
Cues for coaching athletes during the COVID-19 second wave
}

\author{
Silvia Teodorescu ${ }^{\mathrm{a}}$, Frederic Dutheil ${ }^{\mathrm{b}, \mathrm{c}}$ and Constanta Urzeala ${ }^{\mathrm{d}, *}$ \\ ${ }^{a}$ Doctoral School, National University of Physical Education and Sports from Bucharest, Romania \\ ${ }^{\mathrm{b}}$ Université Clermont Auvergne, CNRS, LaPSCo, Physiological and Psychosocial Stress, University Hospital of \\ Clermont-Ferrand, CHU Clermont-Ferrand, Preventive and Occupational Medicine, WittyFit, Clermont-Ferrand, \\ France \\ ${ }^{\mathrm{c}}$ Faculty of Health, School of Exercise Science, Australian Catholic University, Melbourne, Victoria, Australia \\ ${ }^{\mathrm{d}}$ Sports and Motor Performance Department, National University of Physical Education and Sports from Bucharest, \\ Romania
}

Received 28 September 2020

Accepted 28 September 2020

Keywords: Covid 19, athletes, second wave

\section{Introduction}

Given the alarming number of cases reported daily [1] sportsmen need to be prepared for a second wave of COVID-19. The first lockdown definitely found athletes, coaches and the entire staff unprepared. The extension of bans on training under normal conditions, the suspension of competitions and the uncertainty regarding the time of resuming training activities had a negative impact on sports. The involved actors hardly handled online workouts, athletes did their best to practice in narrow spaces or outdoors, coaches struggled with technology, and managers asked for reports. To fight future similar effects, in case of the COVID-19 second wave, we propose several dimensions of the lockdown preparation, starting from a coaching model with four components:

\footnotetext{
*Corresponding author: Constanta Urzeala, Department of Sports and Motor Performance, National University of Physical Education and Sports from Bucharest, 140 Constantin Noica, 6 District, Bucharest 060057, Romania. E-mail: constanta.urzeala@unefs.ro.
}

Problem identification - the overall goal will be to maintain each athlete's general and specific physical fitness

Where the practice conditions allow it and the specificity of the sport requires it, technical, tactical, and artistic training objectives will be established as well. Regardless of the sport, psychological training objectives will also be set. The training goals will be individualised depending on athletes' level of training, gaps and home practice conditions. We recommend: keeping physical preparation at the forefront of lockdown training; rehearsing technical elements if home settings allow; pay attention to the difficulty of technical skills performed by athletes alone, especially at a young age, to prevent injury; examining personal routines from previous competitions and identifying the possibilities to increase their value; studying other competitors; performing psychological preparation with a specialist [2] athletes at the end of their careers, whose performance goal was to participate in the Tokyo Olympics, will be a vulnerable population; in their case, mitigating the 
negative impact of the Olympics postponement will be pursued; athlete withdrawal from performance activity remain a major risk; providing athletes with specialised diet assistance by complying with both the nutritional needs and the somatic requirements demanded in certain sports; specialised federations and sports clubs will get involved [3] and provide equipment, apparatus, audio-visual aids, etc; the training schedule will be kept for the athlete not lose the habit of practicing at a certain time of the day; for young athletes, online training will be correlated with online school; this training stage should be approached as an opportunity [4] to compensate for certain gaps or to allow time for medical recovery.

\section{Action - planning and implementing lockdown training}

Regarding the periodisation of training, online sessions won't represent a transition period, but a basic preparation stage including: planning a mesocycle to maintain the performance capacity that can be performed at home; individualisation of means; giving accurate information about exercises and effort settings, depending on available resources; drawing up training programme models by the specialised federations, as resources for coaches; issuing challenges between athletes but also between the athlete and the coach to support motivation; keeping a diary where the athlete records the content of training and diet; individual documentation of athletes and coaches on the latest training methods; teambuilding exercises, especially for athletes who perform team/group sports; involving athletes in charity/sport promotion projects to nurture their sense of purpose; proposing them personal development activities; catching up educational gaps; dualcareer counselling; identifying the athletes who may have chances to qualify for the Tokyo Olympics, depending on changes in the qualification system due to the COVID-19 crisis.

\section{Assessment}

First step is to check the realization and fulfilment of the training goals. In online training, an initial assessment will be performed to identify as precisely as possible the baseline of the preparation during the new mesoclycle. Athletes will get used to build their selfassessing skills. Monitoring the preparation will never stop.

\section{Feedback}

We recommend providing positive and constructive feedback, given the mental state of both the athlete and coach as a result of training restrictions, postponement of performance goals and fear of catching the disease or transmitting it.

Coaches need to master electronic devices in order to have close communication with their athletes. The gap between the athletes' IT skills and the coach's poor knowledge of technology may negatively affect the training.

The interruption of the specific activity should not be regarded as a holiday but as a continuation of the preparation, with the goal of maintaining the training adaptations and the motivation. Only in this way will it be possible for athletes to resume their competitive activity and be safe from injury, and for coaches, to find internal resources so that, in the future, they can start over.

The COVID-19 second wave should firstly mean responsibility and motivation, but also entertainment and cognitive arousal. Athletes need to feel that they are more than lottery winners at the Olympics for coaches and managers.

\section{Conflict of interest}

The authors declare no conflict of interest.

\section{References}

[1] World Health Organisation [Internet]. 2020 [cited 2020 July 27]. Coronavirus disease 2019 (COVID-19) Situation Report -69. Available from: https://www.who.int/docs/defaultsource/coronaviruse/situation-reports/20200329-sitrep-69covid-19.pdf?sfvrsn=8d6620fa_8.

[2] Toresdahl BG, Asif IM. Coronavirus Disease 2019 (COVID19): Considerations for the Competitive Athlete. Sports Health 2020; 12(3): 221-224.

[3] Timpka T. Sports Health During the SARS-Cov-2 Pandemic. Sports Medicine. 2020; 50(8): 1413-1416.

[4] Jukic I, Calleja-González J, Cos F, Cuzzolin F, Olmo J, Terrados $\mathrm{N}$, et al. Strategies and Solutions for Team Sports Athletes in Isolation due to COVID-19. Sports. 2020; 8(4): 56. 